Tras las huellas del althusserianismo teórico. Una aproximación a la obra teórica de Raúl Olmedo

Jaime Ortega

páginas / año 13 - n 32 Mayo-Agosto / ISSN 1851-992X/ 2021

http://revistapaginas.unr.edu.ar/index.php/RevPaginas

DOI: $10.35305 /$ rp.v13i32.498

\title{
Tras las huellas del althusserianismo teórico. Una aproximación a la obra teórica de Raúl Olmedo
}

\section{In the footsteps of theoretical Althusserianism. An approach to the theoretical work of Raúl Olmedo}

\author{
Jaime Ortega \\ Universidad Autónoma Metropolitana (México) \\ jaime_ortega83@hotmail.com \\ https://orcid.org/0000-0002-8582-1216
}

\section{Resumen}

Este texto abordará la dimensión teórica de la obra de Raúl Olmedo. Profesor mexicano que en la década de 1970 irrumpió en el espacio público a partir del desarrollo de las tesis "althusserianas" que tocaban directamente al marxismo e indirectamente a la teoría de la ciencia. La intervención intelectual de Olmedo lo coloca en un registro amplio -el que se ve envuelto en el marxismo- y al mismo tiempo le otorga un lugar privilegiado, pues, además de ser estudiante con el filósofo Louis Althusser en Francia, el lugar que ocupaba le permitió desarrollarse en números espacios académicos y públicos. Rastrearemos el hilo que va desde sus primeras intervenciones en el campo teórico, pasando por el esplendor "althusseriano" y finiquitaremos en los últimos recovecos de su obra, que vuelven sobre ciertas temáticas.

\section{Palabras Clave}

Teoría; Althusser; marxismo; ciencias sociales.

\begin{abstract}
This text will address the theoretical dimension of Raúl Olmedo's work. Mexican professor who in the 1970s burst into public space from the development of "Althusserian" theses that directly touched Marxism and indirectly the theory of science. Olmedo's intellectual intervention places him in a wide register -the one that is involved in Marxism- and at the same time gives him a privileged place, because, in addition to being a student with the philosopher Louis Althusser in France, the place that It allowed academic and public spaces to develop. We will trace the thread that goes from his first interventions in the theoretical field, passing through the "Althusserian" splendor and we will finish in the last recesses of his work, which return to certain themes.
\end{abstract}

\section{Keywords}

theory, Althusser, Marxism, social sciences

Esta obra está sujeta a la Licencia Reconocimiento-NoComercial-CompartirIgual 4.0 Internacional de Creative Commons. http://creativecommons.org/licenses/by-nc-sa/4.0/ 
Raúl Olmedo es un autor que ha labrado una amplia trayectoria académica, acompañada con una obra escrita significativa. Ella, ocupa diversos campos del conocimiento humano. En la rama principal, que es la ciencia política, se avocó al estudió del municipalismo y la administración pública. En el de nuestro interés: el de una cierta visión de la teoría marxista. Ciertamente no se trata de lo que se podría denominar un marxista clásico, es por ello qué no sorprende la ausencia de una reflexión sobre su producción en el libro de Carlos Illades (2018), hasta ahora el único recorrido que se ha hecho sobre esta corriente en México. Ello es entendible en la medida que se trata de una producción en donde el conocimiento, diálogo y producción a partir del marxismo no lo vincula directamente con el horizonte dominante de éste. Es decir, hace parte de la producción que giró en torno a Marx y los marxismos, pero no se encuentra en las coordenadas fundamentales de las discusiones de la izquierda ni universitaria ni extra-universitaria.

El texto que ahora presentamos busca reflexionar sobre la especificidad de la obra teórica de Olmedo, es decir, el segmento que lo vincula claramente con la tradición marxista y específicamente con el denominado "althusserianismo". Se trata de un ejercicio dentro de los parámetros de la historia intelectual, que opera sobre las reflexiones del autor, los debates que sostiene y las salidas que estas tuvieron en referencia a diversos contextos de discusión. El conjunto de proposiciones que Olmedo sostuvo en el campo teórico lo ubican en el entramado de influencia, esparcimiento y declive del marxismo como opción político-intelectual. Dicha opción se volvió muy común entre la intelectualidad de la segunda mitad del siglo $\mathrm{XX}$, llegando a traspasar, en su influencia, tanto las murallas disciplinares (de la ciencia política a la filosofía) como algunas fronteras políticas. Es el caso de Olmedo, que, sin pertenecer propiamente a la estela de la "inteligencia marxista", es decir, de la que podría identificarse con la izquierda, sostuvo una producción que lo coloca en un diálogo obligado con ella.

Este diálogo se realizó en buena medida a partir de una de las opciones que tuvo el campo intelectual en la segunda mitad del siglo XX: los trabajos del filósofo francés Louis Althusser. Contrario a lo que ha señalado un cierto sentido común, sobre la centralidad de una figura como la de Marta Harnecker, autora del manual Conceptos elementales del materialismo histórico, la influencia y presencia de Althusser se dio a través de múltiples ramificaciones, que incluyen un caudal muy amplio de producciones presentes en toda la geografía latinoamericana.

De tal forma que el texto, al abrevar sobre las formas de operación de la intervención de Olmedo en el campo teórico del marxismo, se desarrollará de la siguiente manera: la primera sección describirá el lugar específico de los estudios sobre la trayectoria de Althusser en América Latina, destacando el lugar que tiene el mexicano; la segunda abordará sus primeros textos, previos a la influencia "althusseriana"; la tercera abordará la centralidad de esta, captando sus enfoques particulares; la cuarta pasará una revisión breve sobre la producción menos conocida del autor, que tuvo un formato periodístico y que opera como bisagra de sus más recientes textos; finalmente, la última, se avocará a sus última producción, después de un periodo 


\section{Tras las huellas del althusserianismo teórico. Una aproximación a la obra teórica de Raúl Olmedo}

largo de ausencia de la teoría, en donde algunos de los temas ya tratados con anterioridad vuelven.

\section{Althusser en México y América Latina}

El día de hoy existe ya un panorama mucho más amplio sobre la recepción, apropiación y crítica de la figura de Althusser en México (Draper, 2017; Pacheco 2017) y América Latina (Rodríguez y Starcenbaum, 2017). La evaluación de dicha situación inicia con la sorpresa confesada en sus memorias sobre el impacto de sus textos fuera de Francia (Althusser, 1992: 312). Al final, Althusser era un pensador que tenía frente a sí un conjunto de discusiones que involucraban una "tradición" y unos referentes muy concretos (por ejemplo, Jean Paul Sartre). Sin embargo, la intervención teórica del filósofo pronto sobre paso los estrechos marcos nacionales, irradió hacia Europa y cruzó el atlántico, extendiéndose de una manera amplia, firme y con no pocos opositores.

En ese cruce atlántico el diálogo inició con la intelectualidad de la Cuba revolucionaria, que lo recibió con beneplácito, pues representó una alternativa frente al marxismo standart exportado de la Unión Soviética. Fue en la isla caribeña en donde se tradujeron muy tempranamente sus textos. Desde 1965, mismo año de la aparición de su primer libro en francés, los cubanos leían ya en español a Althusser (Gómez, 2017). Antes que las ediciones masivas manufacturadas por la Editorial Siglo XXI -cuya traductora fue Marta Harnecker-, en Cuba se produjeron las dos primeras ediciones tanto de los ensayos compilados en los títulos Por Marx (conservando el título de la edición en francés y que posteriormente tomará el nombre de La revolución teórica de Marx) y después en Leer El Capital (nuevamente, conservando el título original en francés y publicando los dos tomos, a diferencia de la edición de siglo XXI). Ambos ejemplares aparecieron en Editorial Revolucionaria, antecedente del Instituto Cubano del Libro. Cabe señalar que los volúmenes, de pasta dura, eran de distribución gratuita.

A partir de la recepción en Cuba, se expandió hacia América Latina con una velocidad vertiginosa. Coincidiendo con el empuje de la "revolución latinoamericana", Althusser fue leído junto a su alumno Regis Debray. Aquella situación permitió un "diálogo" entre algunas corrientes izquierdistas que apelaron a la lucha armada y la obra del filósofo francés. No resulta casual que, en sustitución de Althusser, llegará unas décadas después Antonio Gramsci, pues mientras el francés se asociaba con una lectura filosófica de Mao-Tse-Tung en tiempos de la estrategia armada, el italiano aparecía como el teórico de la democratización. Esto, por supuesto, podría ser matizado de múltiples maneras, pero no queda duda que Althusser fue "hegemónico" en la última parte de la década de 1960 y prácticamente toda la de 1970, es decir, fue el principal motivo productivo, pero también el objeto privilegiado de las críticas. 
Alrededor del continente, sin embargo, la situación se volvió mucho más plural. El así denominado "althusserianismo" trastocó formas ideológicas diversas e incitó a una producción teórica más diversa. Coloquemos algunos ejemplos de ambos registros. En el ámbito político el "althusserianismo" convocó a comunistas como Carlos Cerda o a la izquierda cristiana con Tomás Moulián (2009) y Marta Harnecker en Chile, marxistas más cercanos al nacionalismo popular como Carlos Pereyra (Ortiz, 2001) o militantes de la causa guerrillera como Alberto Híjar (Esquivel, 2015) en México; en Argentina a excomunistas como José Aricó o maoístas como Mauricio Malamud (Malamud, 2017). En términos teóricos convocó a esa misma pluralidad, Híjar lo hizo en la crítica de la práctica artística, Pereyra lo hizo en el seno del análisis de la relación de fuerzas, Enrique González Rojo en el terreno de la epistemología (González Rojo, 1975); en Argentina el principal foco de atención fue el psicoanálisis (Starcenbaum 2017a), en donde diversos autores avanzaron por la veta abierta por Althusser y entroncaron pronto con Jacques Lacan.

Por su parte, las críticas no se hicieron esperar. A Althusser se le acusó de cientificista, revisionista, politicista, ecléctico, inspirador del aventurerismo político y anti humanista. Tanto en Europa como en América Latina la lista de críticos es larga, mencionamos a los más importantes en la región: Adolfo Sánchez Vázquez, Bolívar Echverría y Enrique Dussel en México, Carlos Nelson Coutinho y Florestan Fernandes en Brasil, Javier Sasso en Uruguay, León Rozitnchner en Argentina, Fernando Martínez Heredia y Zaira Rodríguez en Cuba. Todos ellos hicieron parte de un debate intenso que en apenas un par de décadas removió todas las certezas e instaló un campo inédito de discusión dentro del marxismo.

Es legítimo entonces preguntarse por el lugar específico que ocupa Olmedo en este entramado. La primera coordenada que debe ubicarse es que no se le conoce una militancia partidaria con algún grupo de izquierda en la década de 1970, cierto es que, según Octavio Rodríguez Araujo, Olmedo participó junto a Carlos Pereyra, Rolando Cordera, Roberto Escudero y otros, en la fundación de una efímera revista llamada Nueva Izquierda (Rodríguez, 2002) en el primer lustro de la década de 1960. Sin embargo, a su regreso de Francia a principios de la década de 1970 no existe dato del vínculo con algún grupo de esta tendencia política. La segunda coordenada es el lugar que ocupó en el espacio universitario, como otros "althusserianos" fue un profesor e investigador durante buena parte de su vida, vinculado entonces a los devenires de la política universitaria. Finalmente, entre los lectores y seguidores del francés en México, Olmedo fue el único que desarrolló una lectura en clave de "filosofía de la ciencia", como mostraremos más adelante. Esto lo coloca en una línea de desarrollo con escaso diálogo -algo no raro entre los lectores del francés- con sus pares, desarrollando una discusión independiente, sin vínculo directo con otras producciones. 


\section{Tras las huellas del althusserianismo teórico. Una aproximación a la obra teórica de Raúl Olmedo}

\section{Los primeros textos}

En el rastreo que hemos realizado, los primeros textos aparecidos con la firma de Raúl Olmedo que pueden ser comentados, en la medida en la que hacen parte de una cierta constelación, se encuentra en el año 1967 en la revista Comunidad que publicaba la Universidad Iberoamericana. El primer tema que trata es el del racismo contra la población negra en Estados Unidos y comenta la razón por la cual una serie de disturbios comienzan a ocurrir en algunas ciudades norteamericanas. Dice Olmedo "El problema negro tiene sus raíces en la esclavitud, en la explotación y en el fracaso de la reconstrucción del sur de los E.E. U.U después de la guerra civil" (Olmedo, 1967: 364). El autor ensayó una explicación que va de lo sociológico aduciendo las causas del racismo en la estratificación social- a un cierto psicologicismo, en donde el egoísmo, escribió: "se convierte en la condición vital de existencia" (p. 365). Estas dos vertientes alternan en su explicación, tanto de los efectos sociales, como de las actividades del grupo denominado "Ku Kux Klan", como en ciertos comportamientos individuales de los blancos en su relación con la población negra. El cierre del texto merece ser citado, pues expresa los bríos de la escritura del joven politólogo: "Hoy el caliente verano excita al a rebelión negra. El calor no sólo viene del sol ni del clima. Las ondas más cálidas parten de Vietnam y hacer hervir la sangre de color, de todos los colores" (p. 374).

Unos meses después, de nuevo en Comunidad, aparece el primer texto que lo ubicaría dentro de la estela marxista, tanto por la temática como por las referencias. El año 1968 también da cuenta de su primer acercamiento a Althusser, citando ya la edición española de Pour Marx y anunciando la pronta aparición de la traducción al español de Lire Le Capital. Aunque se trata de un texto breve, aparecen ya las reflexiones sobre las intervenciones teóricas de Regi Debray y André Gunder Frank. El objeto es el estudio de la especificidad del capitalismo latinoamericano. Podemos bien identificar que la línea desarrollada en ese momento por Olmedo se relaciona directamente con las llamadas "teorías del subdesarrollo y la dependencia" que comenzaron a ganar terreno. El trabajo ubicó una distinción y diferenciación entre desarrollo y subdesarrollo y de cómo el segundo no es la antesala del primero, en la acepción de Gunder Frank -quien por entonces enseñaba en México-. Olmedo expresa una clara y certera crítica del desarrollismo como ideología abanderada por los grupos dominantes de algunos países e incluso apela a la necesidad del uso de la violencia política y del método guerrillero:

Hemos presentado una visión estructuralista del sistema mundial del capitalismo en la etapa actual. Se esté o no de acuerdo con ella, el hecho es que se ha convertido en la teoría más importante, más debatida, en los medios de la izquierda latinoamericana. Pretende enriquecer al marxismo revolucionario rompiendo con toda una tradición de marxismo momificado y cerrado a las nuevas teorías. Y esta ruptura asume formas vivas, la forma del joven guerrillero, la forma del padre Camilo Torres, la forma de la montaña, la forma de la vida o de la muerte, de la patria o de la muerte (Olmedo, 1968: 17). 
Sin referencias explícitas a Althusser, Olmedo continuó esta línea de trabajo. En el número 18 de la misma revista presentó un ensayo donde criticará las teorías de desarrollo capitalista y mostrándose en simpatía con la que denomina es la "teoría del excedente", es decir, el marxismo de los economistas norteamericanos Paul Sweezy, Paul Baran y nuevamente de André Gunder Frank. El objetivo es la comprensión de las vías de determinación de un tipo de capitalismo específico, el que se desarrolla en la región, no subsumiendo la experiencia particular a una supuesta forma universal. Esta discusión que Olmedo remite a los economistas padres de la teoría del "capital monopolista", tocó profundamente la discusión de la sociología latinoamericana, una década después, cuando los planteamientos de Althusser permitan problematizar la "articulación de modos de producción". Sin duda, este es un primer adelanto que Olmedo hace, aunque todavía sobre la base de un conjunto de referencias que después desaparecerán. Vale la pena, por último, señalar que este ensayo fue publicado en la revista Pensamiento Crítico de Cuba (Olmedo, 1970). Olmedo es el único autor mexicano que fue publicado en aquella importante revista que irrumpió en el espacio intelectual de la revolución cubana. Justamente en la entrada de esa versión, los editores señalan que el texto es útil en la medida en que presenta una síntesis apretada, pero que, por ello mismo, se encuentra limitada en su argumentación.

Como último dato es curioso señalar que en Comunidad apareció un breve ensayo que polemizaba con los planteamientos de Olmedo, hecho por el político y académico boliviano Mario Rolón Anaya (quien tuvo distintos cargos ministeriales y como embajador durante las dictaduras de Barrientos Ovando, Bánzer y García Meza). En dicho texto señala que el estructuralismo que Olmedo representa es el responsable del aventurerismo político que conduce al uso de la violencia social de minorías carismáticas. Un texto breve, sin mucho contenido, pero que expresa bien cómo se observaba una alianza entre los grupos armados y lo que se denomina la concepción "estructuralista", que habría, explica el autor, nacido en Francia para revitalizar el marxismo (Rolón, 1969: 658-662).

\section{La edad de la pasión}

No se trata tanto de hacer una biografía de un individuo, sino rastrear sus intervenciones teóricas al seno de ese campo muy amplio, como fue el marxismo de la segunda mitad del siglo XX. Es por ello qué no hemos insistido en datos específicos sobre la trayectoria de Olmedo. Sin embargo, la época siguiente de su producción está indudablemente marcada por un hecho que fue una experiencia particular: su estancia en Francia. Si bien existe poco registro de su actividad en el país europeo, lo cierto es que algo quedará como efecto: la presencia de Althusser y la vinculación de este nombre como una seña de identidad teórica y política. Como muchos otros latinoamericanos, Olmedo acudió a los cursos y seminarios que el francés dictaba 


\section{Tras las huellas del althusserianismo teórico. Una aproximación a la obra teórica de Raúl Olmedo}

hacia finales de la década, como otros, no se encontraba inscrito en la Escuela Normal, lugar de adscripción del filósofo. Junto al también filósofo Cesáreo Morales, Olmedo es de los pocos mexicanos de los que se conoce que siguieron en vivo aquel espacio de discusión académica-política.

Podemos así, ubicarnos en el periodo posterior a su vuelta de Francia, tras concluir su doctorado en Filosofía con la tesis titulada El estado teórico de la filosofía marxista. Un panorama global de su actividad permite ubicar momentos de su itinerario. Primero con su participación en la segunda época de la revista Historia y Sociedad. En ella adelantará un fragmento del Curso de filosofía para científicos de Althusser, que se encontraba inédito en español. También en sus páginas publicó una carta personal que el filósofo le dirigió, a raíz de que se le enviara un ejemplar de la revista, en la misiva se muestra reticente a aceptar algunas de sus conclusiones (Althusser, 1978). Dirige además un seminario llamado "Leer Materialismo y empiriocriticismo" del cual se publican las ponencias de sus alumnos en la Revista Mexicana de Ciencias Políticas, además de una traducción del texto "Ideología y Aparatos ideológicos de Estado" hecha por Jacqueline Peschard. En ambas revistas Olmedo publica textos variados, sostiene polémicas con diversos académicos y avanza por una línea muy particular del "althusserianismo" mexicano que exploraremos con detenimiento a partir de la síntesis de su obra.

Para realizar una exposición más sintética de la intervención teórica, es necesario referirse a El Antimétodo: introducción a la filosofía marxista (1980), que, de hecho, además de ser su tesis de doctorado es la reunión de los textos publicados dispersamente. Se trata de un total de cinco trabajos que van avanzan desde el tema del materialismo, pasando por lo que él considera es una crítica de V.I Lenin a Federico Engels y sigue hasta las teorizaciones sobre el concepto de modo de producción, además de la exposición del capítulo primero de El Capital, producto de sus clases como encargado del Seminario de El Capital que se desarrollaba en la FCPyS.

Podemos evaluar la intervención de Olmedo en dos dimensiones que aparecen en prácticamente todos sus textos. Por un lado, hay una intención "filosófica" bastante débil con respecto a lo que disciplinarmente se entiende por ella, ya que concentrar sus energías en la oposición materialismo-idealismo y su deriva como proceder metodológico. Por otro, hay un intento de generar una lectura no-dialéctica de la obra de Marx y de Lenin, que resulta un tema mucho más sugerente, aunque esté plagado de lugares comunes de cierta "filosofía marxista". La pugna principal de Olmedo es con aquellos teóricos que consideran al marxismo como una "metametodología", es decir, rebate cualquier intención de construir el método por excelencia. Se trata de un combate con la tradición iniciada por Gyorgy Lukács y el marxismo hegeliano dominante en Europa. Por el contrario, en sintonía con la filosofía de la ciencia francesa de aquel tiempo, aboga por lo opuesto, es decir, que cada ciencia de acuerdo a su objeto determina su propio método. En ese sentido Olmedo busca demostrar que Marx abordó sólo el problema inicial del continente de la ciencia de la historia (es decir lo que tiene que ver con los modos de producción 
y las formaciones sociales) pero de ninguna manera se trata de una "cosmovisión" (como insistían las lecturas de Antonio Gramsci) ni de un "método general" (como lo colocó el marxismo-hegeliano) y mucho menos de una "filosofía" que hablara de todos los temas. Dos son los intentos de desmovilización, por un lado, la pretensión "metodologicista", que ubicaría en Marx el descubrimiento de una nueva de hacer ciencia; pero por el otro, el que el filósofo alemán habría fundado una "nueva filosofía", que tendría que comprender no sólo una epistemología, sino también una ontología, una ética, una estética, una política, es decir, un sistema.

Comenzando por cada uno de los posicionamientos, el texto "El materialismo" con el que se abre El anti-método versa precisamente sobre los dilemas metodológicos. Se trata de un texto que pretende elevarse por encima de las propuestas en boga, pero claramente limitado en sus argumentos, a pesar de los avances que se daban ya con respecto a las corrientes más vulgares del marxismo, Olmedo siguió insistiendo que lo que se dio a llamar el "idealismo" no partía de la "materia", sino de las "ideas" abstractas. En ese sentido Marx habría dado un paso al abordar de manera científica o "materialista" los problemas sociales, no sólo porque partiría de la categoría de "materia", sino por que eliminaría la dicotomía sujeto-objeto propia de las filosofías trascendentales y de la conciencia, así como la herencia especulativa al eliminar la confusión objeto pensado/objeto real. Estos dos últimos problemas son de tomarse en serio, porque con ellos Olmedo rompía gran parte de la tradición política que ubicaba a Marx como un continuador de Hegel o hacer del marxismo la aplicación del método hegeliano a la economía política clásica. La ruptura de esos dos puntos (dicotomía sujeto-objeto y diferenciación entre objeto pensando y objeto real) permitía una nueva concepción que eliminaba de tajo el problema epistemológico clásico de la garantía del conocimiento y la supuesta necesidad empirista de correspondencia entre pensamiento y realidad. Todos los aspectos mencionados serían los que señalarían el rasgo distintivo tanto de las epistemologías idealistas o lo que se llamó en su momento el defecto idealista del materialismo espontáneo. Con este texto Olmedo dejaba sentado la base sobre la cual ejercería una crítica a todo intento de presentar al marxismo como "metamétodo".

El conjunto de problemas planteados inicialmente tuvo continuidad en "El reproche de Lenin a Engels acerca de la dialéctica". Olmedo busca hacer extensiones de las breves críticas que Lenin dirige a Engels a propósito del tema de la dialéctica y la forma de exposición. De pequeñas frases que Olmedo encuentra en las Obras del dirigente político ruso, desprende un largo texto que tiene un núcleo fundamental: desmontar cualquier posibilidad de concebir que la dialéctica heredada por Hegel expresa una opción teórica de producción de conocimiento adecuada para el marxismo. Para el filósofo mexicano tratar de forzar en el proceder científico del marxismo (en el campo de la historia) una propuesta filosófica, obliga a que esta última funcione como procedimiento de construcción de conocimientos y al tiempo como la garantía de la correspondencia del conocimiento con el objeto: la joven ciencia de la historia lo que menos necesita es una filosofía de la historia. Ambos 


\section{Tras las huellas del althusserianismo teórico. Una aproximación a la obra teórica de Raúl Olmedo}

elementos, el del procedimiento universal y el de las garantías serían propios de toda posición idealista, en cambio la posición materialista (que sería en su argumento la postura científica) no estaría preocupada por la correspondencia entre concepto y realidad y comprendería la autonomía total del objeto de pensamiento en su estructura de inteligibilidad. Para decirlo más breve, Olmedo argumento que lo que se construye como categoría científica en la historia tendría su propia lógica, de acuerdo a su objeto de estudio y no a un método predispuesto universalmente. Leyendo los comentarios a Hegel del propio Lenin, Olmedo busca demostrar qué, aunque haya expresiones acordes con la dialéctica hegeliana tanto en las obras del revolucionario ruso como en las de Marx ello no permite afirmar que la dialéctica es parte del universo de la ciencia de la historia. La dialéctica no tendría estatuto científico para Olmedo en tanto que se asienta en elementos idealistas (la ya mencionada dicotomía sujeto-objeto, la confusión entre objeto de pensamiento y objeto real, etc...). Tras el recorrido de la obra de Lenin y contrastando distintos momentos de su obra, escribe:

Larga trayectoria la de Lenin, que es la trayectoria de un retorno necesario a la consigna de Marx y Engels: si se quiere una dialéctica para el marxismo ésta es la dialéctica de Hegel "puesta sobre sus pies", es decir, sin su transformación en método operacional, es decir, como simple metáfora de la historicidad del universo que sirva para combatir visiones estáticas, metafísicas y reduccionarias (sic) de la sociedad (Olmedo, 1980: 51).

Olmedo rechaza entonces la cientificidad (para el estudio de las sociedades) de la dialéctica al afincarse en presupuestos idealistas, que someterían al conocimiento producido a una supuesta comprobación con la realidad, es decir, a una mixtura entre pragmatismo y empirismo. Por el contrario, el asume que es necesario superar cualquier comprensión de la dialéctica como método de conocimiento. Si algo es posible de salvar de la dialéctica es su consideración como una forma de exposición de argumentos, que conviviría junto a otras.

El texto "El hegelianismo y el spinozismo de Marx" que había tenido una versión previa en Historia y Sociedad busca reconstruir el peso que tienen tanto Hegel como Spinoza en el despliegue de los argumentos de la muy comentada Introducción de 1857 de Marx. Para que se observe en que términos se desplegaba el argumento de Olmedo bastará citar dos párrafos, en el primero define, de nuevo, a los opuestos que se encontrarían en disputa en el terreno filosófico: "la tendencia afirma la primacía del pensamiento sobre la realidad (idealismo) y la tendencia que afirma la primacía de la realidad sobre el pensamiento (materialismo)" (Olmedo, 1980:57). Y para zanjar la relación de los tres autores en cuestión: "Marx era hegeliano en aquello que Hegel era spinozista. Hegel había desarrollado hasta sus últimas consecuencias la fórmula la determinación es negación y había hecho de ella la clave de su obra" (Olmedo, 1980:62). En una operación sumamente extraña Olmedo sostiene que Marx abre "dos frentes" de lucha teórica en el texto fechado en 1857. Por un lado, abre el "frente Spinoza" para evitar que el método particular de la ciencia de la historia se vuelva método general de toda ciencia. En tanto que el 


\section{Jaime Ortega}

"frente Hegel" es el que bloquea el paso de la dialéctica como propiedad de la materia a un "método" para producir nuevos conocimientos. Como hemos señalado anteriormente, Olmedo sostenía que lo que se podía entender por dialéctica era una forma de exposición ordenada de los conceptos, pero que estos se habían producido al seno de la ciencia de la historia fundada por Marx, es decir, que la dialéctica no producía nuevos conocimientos. Sin duda es un texto más sugerente que los anteriores, en tanto que formula una hipótesis que se encontraba, hasta ese momento, ausente, que es la presencia de Spinoza y no sólo de Hegel en la obra de Marx, aunque la forma en que opera sobre ambos autores y el propio Marx resulta algo sorpresiva.

Siguiendo con su argumentación el siguiente texto se titula "El primer capítulo de $E l$ Capital", el más interesante de todos. La tónica se encuentra ya delineada por los anteriores trabajos: atacar cualquier pretensión de extraer de tal o cual obra de Marx un método general, así como la intención de desmovilizar cualquier intento de hacer del marxismo una nueva filosofía cuyo núcleo sería una noción de dialéctica (hegeliana o de otro tipo). Para Olmedo el capítulo primero de El Capital no es "el más dialéctico" como se suele decir, pues en el subyace una lógica formal básica (expresada en las distintas formas del valor, que el autor explica en términos de funciones equivalentes y relativas). El componente supuestamente "dialéctico" se encontraría en la forma de la exposición, a partir del concepto de valor, que involucra temáticas como la oposición, la identidad, la transformación de un término a otro, de lo cuantitativo a lo cualitativo, para el autor: "dos exposiciones simultáneas, pero absolutamente independientes" (Olmedo, 1980: 87). La exposición "formal” la encuentra Olmedo en el desarrollo lógico de la ecuación de la primera forma, la que se denomina "simple" del valor $(A=f(B)$,) a través de cinco momentos en los que se introducen condiciones provenientes del intercambio social para quien juega el papel de (B), es decir, del equivalente. La supuesta "dialéctica" del capítulo primero sería una ficción, el resultado de una confusión de términos, a los que Olmedo se dedica a atacar en la última parte del texto. En ella embiste contra las ambigüedades del uso del término dialéctica, que tendría acepciones encontradas, como ser una propiedad de la materia o un método general, pero para otros es, además, una visión del mundo: todos esos usos del concepto, impiden, observar la "dialéctica" en el capítulo primero de El Capital, que está ceñida, para Olmedo a nociones básicas de lógica formal. Todo ello demuestra como la ciencia social es contenedora de métodos que pugnan por imponerse los unos a los otros, según el autor, razón última para entender la lucha de clases en la teoría.

Una vez expuestos en sus núcleos centrales los textos, podemos profundizar en el vínculo que estas posiciones tenían con Althusser. Lo primero que habría que decir es que más allá de que este des autorizara el lenguaje de Olmedo, según consta en una carta que se publicó en Historia y Sociedad, lo cierto es que el mexicano era un "althusseriano" en forma. Si comparábamos su actitud con respecto a otros, como González Rojo o Pereyra, quienes realizaron críticas agudas al francés, o la de Híjar, 


\section{Tras las huellas del althusserianismo teórico. Una aproximación a la obra teórica de Raúl Olmedo}

que mantuvo una línea ecléctica al colocar en diálogo esa filosofía con la de Herbert Marcuse, es perceptible en la lectura, una línea más dura de argumentación.

Lo segundo que hay que señalar es que Olmedo desarrolla sus trabajos en correspondencia con las posiciones de Althusser en su auto crítica, en donde se da vuelta al teoricismo y se da paso a una mayor dimensión política. En toda la argumentación del mexicano subyace la idea de que la filosofía es un campo de batalla teórico, en donde no hay propiamente filosofías independientes, sino posiciones dentro de ella. Así, materialismo e idealismo, serían en realidad dos posiciones al seno de toda filosofía y esta, a su vez está representada en la lucha de clases (Althusser, 1970: 73).

Althusser sostuvo, desde los textos compilados en La revolución teórica de Marx una disputa con el marxismo que afirmaba que este era un método de conocimiento. A ello opuso la idea de la práctica teórica, que contemplaba un esquema parecido al ofrecido por Marx en el capítulo quinto de El Capital. Al igual que el obrero que fabricaba una mercancía a partir de la disposición de materia prima e instrumentos de trabajo, el teórico elaboraba un conocimiento nuevo a partir de percepciones y sensaciones que son modificadas en la utilización de conceptos y teorías. Olmedo no desarrolla este último tema, pero si el primero.

Al igual que el francés, hay una obsesión por el tema de la dialéctica. Podríamos decir que sigue siendo un terreno de disputa candente. Mientras que Lukács y la estirpe que le siguió insistió en una deriva donde Marx y Hegel tenían que ser comprendidos como continuidades, Althusser rechazó esta hipótesis a partir del concepto de ruptura epistemológica. Este tema quedó superado en su postura cuando abandonó la idea de formalizar una "dialéctica específicamente marxista" y renunció, definitivamente, cualquier idea de dialéctica. De lo que se trataba era de abandonar toda forma dialéctica, porque ella restauraba, necesariamente, una concepción idealista y esta conducía a reestablecer supuestos que se consideraban superados, como lo eran una filosofía de la historia, una visión especulativa que no separaba objeto de conocimiento y objeto real, principalmente. La dialéctica tenía que ser expulsada del marxismo y el texto de 1857 de Marx servía para ello, pues el francés leía que en ella se rompía toda forma especulativa que equiparara el objeto real al objeto de conocimiento, punto clave para él. Es muy claro que Olmedo retoma esta postura y la lleva al extremo.

\section{Intermedio: El Capital en el espacio público}

Hacia finales de la década de 1970 Olmedo comenzó a variar sus temas de escritura. En el diario Excelsior escribió regularmente una columna en donde sigue la coyuntura económica. Esas notas fueron recuperadas, parcialmente, en un libro (Olmedo, 1983). Sin embargo, nos resulta sugerente pensar una serie de textos que el autor dedicó al centenario de Marx en el año 1983 dentro de su colaboración en dicho periódico. Es relevante por dos motivos, primero, la presencia de Marx en el 
espacio público, a través del periodismo, y el segundo, que estos textos forman una bisagra entre su producción "althusseriana" previa y lo que vendrá después.

Se trata de 13 publicaciones, que van del 14 de marzo (justo en el centenario del nacimiento de Marx) al 2 de abril. En ellas explicita una visión distinta del marxismo. Es un intento pedagógico, al tiempo que pueden leerse como alternativas de la interpretación "althusseriana" que sostenía en sus ensayos académico. Su actividad como columnista es una grieta en esta interpretación, pues permite observar su lectura propia, más allá del canon establecido por el francés.

En la primera nota (1983a $)$, por ejemplo, sostuvo que al igual que la Biblia, las ideas de Marx son inspiradores de una "visión del mundo". Coloca al marxismo, como a las ideas desprendidas de la Biblia, por fuera de la dicotomía "verdad-falsedad", lo cual deja al marxismo por fuera de los cambios técnicos o políticos que sucedían en la sociedad. En la segunda (1983b) colaboración habló de la riqueza y la forma que esta había adoptado, diferenciado entre el feudalismo y el capitalismo, llegando a la conclusión de que hay una "ley histórica", que es la distribución de la riqueza, que cambia según el periodo histórico. La tercera (1983c) columna explicó el concepto de lucha de clases de una manera muy sintética, sin embargo, destacó hacia el final la contradicción ecológica que tiene la industrialización. De este elemento desprendió que el proletariado debería de anteponer los intereses humanos en general antes que los de clase, refiriendo a la obra de Rudolf Bahro como una clave de esta problemática que cruza la clase y el "holocausto" ambiental. La cuarta (1983d) entrega habló de la fuerza de trabajo, explicando la centralidad de la venta de las capacidades de trabajo, es decir, la forma más importante de la mercantilización. Resulta crucial que Olmedo argumentó que en las sociedades socialistas (es decir, la URSS y demás países europeos) seguía dándose la compraventa de la fuerza de trabajo como mecanismo atravesado por la presencia del Estado. En esa línea, la siguiente columna (1983e) expuso que existía una correlación de fuerzas políticas entre el comprador y el vendedor de la fuerza de trabajo y que esta se expresaba en el nivel salarial. La entrega siguiente desarrolló el nivel "psicológico" de la condición de vendedor de la fuerza de trabajo, argumento que las teorías de la alienación y el fetichismo son teorías psicológicas que Marx aportó para comprender el comportamiento humano.

Después de estas primeras entregas, Olmedo comenzó a entrar a explicar algunos conceptos (1983f). El primero es el de la teoría del valor-trabajo, que presupone la equivalencia de las mercancías. Después da un paso arriesgado (1983g), al criticar el uso de los conceptos valor de cambio y valor de uso al referirse a las mercancías. Desde su punto de vista Marx se equivocó al usar el concepto valor de uso y, esto se corrigió cuando se comenzó a hablar de utilidad. El tono de su siguiente columna (1983h), dedicada a la división del trabajo es más que una explicación de esa situación social, una lectura crítica de los usos que Marx hizo de ella. Según Olmedo para el joven Marx la división social del trabajo fue el origen de la propiedad privada, por lo tanto, al eliminar una se disolvía la otra; en cambio, en su madurez, Marx habría corregido esto, entendiendo que se podía acabar con la propiedad privada y 


\section{Tras las huellas del althusserianismo teórico. Una aproximación a la obra teórica de Raúl Olmedo}

conservar la división del trabajo. De nuevo, continuando con un tono polémico (1983i), Olmedo argumentó que quienes ven en el trabajo el origen del valor (o del valor de cambio), se equivocaban, ya que el alemán habría criticado esta tesis, pues en su interpretación el valor de cambio solo aparece en la distribución. Así, dando continuidad a esta última tesis, la columna aventura más adelante (1983j), que la posibilidad de la equivalencia, es decir, de encontrar un valor de cambio universal, sólo se encuentra en la distribución y que esta depende de mecanismos de negociación y de poder. La penúltima (1983k) entrega de su columna redondeó este argumento: la teoría del valor no se afinca en la producción, sino sobre la distribución y en ese plano es más que una teoría económica: es en realidad una teoría política. Así, el valor se determina en el mercado (1983l) y no en el tiempo de trabajo socialmente necesario para producir un bien. La última de sus columnas de esta serie criticó a las teorías del intercambio desigual (1983m), pues consideran que el valor es algo inherente al producto y no un atributo de poder que se decide en el intercambio real, es decir, en el mercado.

Hasta aquí podemos señalar tres elementos que nos parecen fundamentales. El primero es que se trató de un esfuerzo pedagógico, limitado espacialmente, por tanto, es claro que muchas de las ideas están concentradas y expuestas de manera tajante, sin mediaciones o posibilidades de ampliarse, motivo por el cual decidimos tratarla por aparte y no junto al conjunto de los ensayos académicos. El segundo es que se trató, en pleno, de una exposición sobre temáticas que Marx abordó en El Capital y no de la interpretación meta-teórica que fue clave en los análisis de Althusser. Es ahí donde se muestran las cuestiones más candentes de su producción, cuando por ejemplo, pone énfasis exclusivo en la distribución, sin mencionar jamás la producción, sin embargo, esto es más que un error interpretación, sino en realidad a una forma de producir a partir del texto clásico. La teoría del valor-trabajo no es substancialista (es decir, el valor no existe por fuera del intercambio) y al dar tanto énfasis al vínculo entre mercado y poder, inaugura una veta original, una proposición que no era fácil encontrar en Althusser. Finalmente, es que en estas exposiciones hay puntos muy finos en donde su interpretación se separa de la del francés, a diferencia de los ensayos académicos donde busca mantener una línea más rígida en concordancia con él. Por ejemplo, hablar de "leyes de la historia" en la filosofía de Althusser resulta algo inconcebible, lo mismo que referirse a Marx como autor de conceptos psicológicos.

\section{Pasos adelante}

Olmedo se retiró de la producción en torno a la teoría a mediados de la década de 1980, cuando su actividad profesional se enfocó a desarrollar con mucho detenimiento, el problema de la municipalización y del poder "comunitario." Algunas de sus producciones en esa época aparecían en una editorial llamada "Comuna", y en general, evaluaba situaciones de orden de gobierno, aunque también 
se dio el primer caso de su recuperación de ensayos de décadas previas, también apareció un ensayo a propósito del conflicto universitario de 1999 (Olmedo, 2001). Evaluemos ahora el último segmento de su producción, que contiene novedades y también reiteraciones. A lo largo de la última década Olmedo publicó cuatro libros: Democracia: máquina tautológica que produce deseo y placer de dominar y de someterse (2013), Democracia es religión (2015), Las democracias perdidas (2016) y Violencia es religión: los mecanismos de pensamiento que originan las violencias (2016). La tetralogía expresa un decidido y firme proyecto, en tanto que recupera recovecos de su pasada producción teórica, pero también pasos adelante. Dado que el espacio es limitado y no es nuestro objetivo desentrañar todas las discusiones sobre las que se posiciona, procederemos de la siguiente forma: expondremos las novedades que contienen esta tetralogía para después confirmar aquellas trayectorias comunes con la producción previa.

Existen una serie de novedades en los planteamientos de Olmedo, que podemos sintetizar a partir de una noción básica: la crítica de la democracia, en el capitalismo, como una forma perversa de dominación. Es esto lo que desarrolla de manera central el volumen Democracia: máquina tautológica... Con este texto se abre, sin duda, un capítulo novedoso en su reflexión. Es notorio que en el hay una utilización de autores que habían estado ocultos -aunque quizá no ausentes- de sus reflexiones previas. En primer lugar, Friederich Nietzsche es interpretado como un "materialista histórico" en tanto que hizo crítica de la aparición de la deuda. Emergen otros autores de cierta tradición francesa como Emile Durkheim, George Bataille, Gilles Deleuze y Félix Guattari, quienes mostraron su firme presencia en el recorrido de la obra. El argumento, sintetizado, es que el capitalismo demandó una forma de sometimiento de los seres humanos, que es la democracia, en la cual no se hace sino desarrollar un goce por el sometimiento a partir de la subjetividad que reproduce la identidad de lo mismo, es decir, de equivalentes, que encuentran su dato más inmediato en el intercambio mercantil.

Sin embargo, y esto es importante, el argumento crítico sobre la democracia que Olmedo empezará a desarrollar viene acompañado, pues se engancha con segmentos previos de su producción. Aparece así una seria continuidad de algunas de las preocupaciones, por ejemplo, es muy claro que en su argumento de la democracia como una "máquina tautológica", se conecta mucho con lo expuesto en sus columnas periodísticas. Lo que ahí apareció como un destello de lectura directa de Marx, aquí se presenta como un eslabón fundamental: es en el problema de la equivalencia en donde se genera una totalidad que genera identificaciones de subordinación. Es decir, en el mercado y el intercambio no sólo se generan equivalentes entre objetos, sino una subjetividad que reproduce una identidad. La democracia es el medio preferido del capitalismo porque es la expresión política de la equivalencia, que vuelve a los seres humanos abstractos, idénticos.

Antes de recorrer algunos de los argumentos principales de Olmedo en cada una de estas obras, nos permitimos señalar los puntos, no sólo argumentales, sino también de composición de las obras, que lo vinculan con el periodo de producción a rededor 


\section{Tras las huellas del althusserianismo teórico. Una aproximación a la obra teórica de Raúl Olmedo}

de Althusser y el marxismo. Por ejemplo, Las democracias perdidas contiene textos que fueron redactados en épocas previas. El ejemplo más claro es un acápite dedicado a Charles Bettelheim que había aparecido en un estudio que recorría distintas teorías del imperialismo. Lo mismo sucede con su análisis crítico de la noción de discurso político de Michel Plon que había sido parte de un importante seminario organizado en la UNAM (1980). Otro tanto se expresa en Democracia es religión, en donde se recoge la intervención que Olmedo había hecho en su seminario sobre Materialismo y empiriocriticismo y que había tenido una exposición temprana en la Revista Mexicana de Ciencias Políticas y Sociales en la década de 1970. Sin embargo, a pesar de que estos textos habían sido previamente publicados, no debe considerarse que su inclusión en estos nuevos libros es un simple ánimo de republicar. Por el contrario, se trata de textos que adquieren sentido tanto en el conjunto de cada texto, como en el del proyecto de crítica de la democracia que Olmedo pretende realizar.

Comencemos por Democracia: máquina tautológica..., sin duda el texto más original, el más logrado y el más amplio. En el centro de la discusión está el concepto de equivalencia, que remite a la igualdad de lo mismo, mecanismo fundamental del intercambio de mercancías. Así: "La tautología y la identificación son las armas más poderosas de la lucha de clases bajo su forma ideológica y conceptual. Tautología e identificación constituyen el oxígeno de la lucha de clases ideológica" (Olmedo, 2013: 73). La equivalencia funda jerarquías que se expresan en la economía, en la pulsión erótica y en la plusvalía sexual. Todo esto lo remite a su lectura de Marx: "Sabemos que el propio Marx dio un nombre a la máquina tautológica (el "fetichismo de la mercancía") y que desde su juventud aprendió de Feuerbach a desmontar su máquina. Su Crítica a la filosofía del Derecho de Hegel, escrita a los veinticinco años (1843), es la denuncia de la forma en que Hegel aplica la tautología para servir al poder del Estado Prusiano, que lo ha elegido como su filósofo oficial" (p. 97). Como se puede ver, en este texto se concentran el conjunto de lecturas, tanto clásicas -con Marx a la cabeza- como novedosas.

Por su parte, Democracia es religión es quizá el texto que más conecta con el lenguaje y los formatos previos de su producción. Se trata de un texto que combina la crítica política con la crítica de todo intento de formular una epistemología. Comienza por una exposición que retoma parte de Democracia: máquina tautológica..., en la medida en la que hace crítica de la equivalencia (definida como igualdad de lo mismo), la libertad y la igualdad como una lógica que en realidad produce desigualdad. Después expone la contradicción materialismo-empiriocriticismo, en donde, Lenin de por medio, realiza un ejercicio de asedio a todo intento de conocimiento general que se base en la dialéctica, es decir, vuelve al tema de la filosofía de la ciencia: la imposibilidad de generar un método general en donde un "sujeto" conoce de manera abstracta a cualquier "objeto", aplicando una "metodología", que es la garantía de producción de conocimiento por muy diferente que sea el objeto al que se le aplique. Esta propensión es muy generaliza porque considera que la dialéctica es un artificio del poder existente. Después, analiza las 
condiciones políticas después de la caída del socialismo real de 1989, llegando a la conclusión de que la verdadera contradicción es la de los modos de producción de tipo industrial versus los modos de producción no industriales, dejando ver la impronta del de-crecimiento que anima esta parte de su producción. Finalmente, insiste en la lectura de Materialismo y empiriocriticismo de Lenin, en las que a través de 51 "Tesis" (aquí, indudablemente tiene una deuda con Althusser), va desde la definición tanto de materialismo como del idealismo, hasta el cómo funcionan la pluralidad de las ideologías dentro de la filosofía. Se trata de un texto breve, pero denso, en la medida en que supone estar consciente de lo que el autor significa con algunas categorías y que, en gran medida, sólo es posible entender si se sigue el curso de su trayectoria. La conclusión es clara: el materialismo atiende a la determinación objetiva, de la situación concreta, en tanto que el idealismo siempre aspira a grandes generalizaciones y la formación de teorías del conocimiento universales y des-localizadas.

El desarrollo de algunas ideas previas continúa en Las democracias perdidas (2016). Se trata de un ejemplar bastante significativo. Tres son sus argumentos. En términos de continuidad ya hemos señalado la presencia de Bettelheim. En ese rubro se desarrolla un argumento que quiere descentrar el primado de las fuerzas productivas sobre las relaciones de producción. Siguiendo al economista francés, Olmedo coloca el énfasis revolucionario en esta perspectiva, que habría sido llevada a cabo por Mao-Tse-Tung, quién al no poseer fuerzas productivas en la china posrevolucionaria, apostó por el despliegue de las relaciones de producción: se trataba de "política que tiene como finalidad la lucha por la producción" (Olmedo, 2016: 16). Este tema había sido tratado con anterioridad, pero colocada en este libro adquiere un sentido pleno, pues viene acompañado de otros argumentos. El segundo tema que tiene continuidad, pero que se reformulación de sus críticas a la dialéctica, que, para él, es la expresión más prístina, en el pensamiento, del desarrollo capitalista. La dialéctica es "un proceso donde los elementos dispersos son unificados y sintetizados en una unidad totalizadora cualitativamente superior a los elementos individuales" (Olmedo, 2016: 17). Ello adquiere dramatismo cuando se vuelve una perspectiva ontológica que adquiere una dimensión política, la dialéctica "es una ideología de Estado; es decir, una ideología de dominación" (Olmedo, 2016: 21) y que adquiere cabalidad a través de tres procesos: la centralización, la concentración y la acumulación. Esta triada es la que coloca como equivalente a la negación, negación de la negación, superación-conservación. Así, estos tres elementos constitutivos en su definición de dialéctica son, en realidad, los del capitalismo. Finalmente, hace un recorrido de temas que en la década de 1970 eran actuales, como lo era el eurocomunismo, el papel de los intelectuales o la presencia cada vez más intensa de la reflexión de Gramsci; de entre ellas destaca el balance que hace de la obra de Althusser. Entrega sobre el filósofo francés una breve reseña biográfica, pero, sobre todo, argumenta que las disputas filosóficas en torno a sus ideas son un callejón sin salida, pues su obra no es filosófica, sino política. Ello lo lleva a criticar los nombres que adquirieron masivamente sus obras: "Lire Le Capital 


\section{Tras las huellas del althusserianismo teórico. Una aproximación a la obra teórica de Raúl Olmedo}

(iLeed El Capital!) fue despojado de su intención de consigna teórica-política y convertido en un académico e indefenso Para leer El Capital, que sugiera el nombre de cualquier manual... (Olmedo, 2016: 115). En tanto que "Pour Marx (que tiene el sentido de ¡Tomemos partido por Marx!) fue maquillado y transformado en un bello momento pretérito de la historia de la filosofía: La revolución teórica de Marx" (Olmedo, 2016: 115).

Finalmente, el texto más despegado de la etapa previa de producción es Violencia es religión (2016 ${ }^{\mathrm{a}}$ ) en donde se desarrollan las tesis contenidas en Democracia, máquina tautológica... (2013). Se trata, para Olmedo, más influido por Walter Benjamin, Franz Hinkelammert e Iván Ilich, del carácter de "contra productividad violenta" (p. 15) de la democracia. Se conecta con el libro de 2013 porque se define que la violencia actúa como religión: "porque violencia es generadora de identidad o identificaciones de grupos sociales, que conduce a delimitar territorios, razas, intereses" (p. 28). El texto de Olmedo ocupa un lugar pequeño en el libro, pues se recolectan algunos ensayos de Pierre Chaunu, Jean Meyer y un conocido texto de Walter Benjamin sobre la violencia. Hacia el final, Olmedo realiza un ejercicio de lectura de Nietzsche, cuestión que no estaba presente en su anterior producción alrededor del marxismo.

\section{Consideraciones finales}

Raúl Olmedo no ocupa un lugar central en las referencias que se suelen hacer al marxismo. El campo en el que más se la he reconocido es el de administración pública, debido a sus aportes en la problemática del municipio como forma de gobierno. Sin embargo, el rastreo que se puede hacer de la bibliografía de la década de 1970 lo coloca en las principales discusiones de esa década, en lo que respecta al marxismo. Esta es, sin duda, una situación paradójica. Aquí hemos tratado de seguir la huella de Olmedo dentro del marxismo y las huellas de Althusser en su más reciente y creciente producción.

Ello ha redondeado en la exposición de sus primeros textos, en el periodo inmediatamente posterior a su titulación como politólogo con la tesis "Génesis del poder" dirigida por Pablo González Casanova. En este primer segmento se muestra una preocupación social indiscutible y su contacto con determinados autores que harán parte de la tradición estructuralista.

El segundo momento es el de la discusión marxista, tras su regreso de Francia y el empeño en defender una posición teórica muy particular, que seguía a Althusser, pero se enfocaba en un combate contra ciertas tendencias, por cierto, ninguna de ellas con mucha fuerza en el campo intelectual mexicano de la época. Olmedo sostiene una posición al interior del marxismo en la que rechaza que a partir de las proposiciones de Marx se justifique una "dialéctica" como herramienta productora de conocimientos. Todo lo contrario, el materialismo consistiría en desmovilizar cualquiera intento de formación de una epistemología. Este segundo momento se 


\section{Jaime Ortega}

amplía, a uno tercero, que hemos analizado a partir de las notas periodísticas dedicas a Marx que Olmedo publicó en 1983. A pesar de su brevedad, en ellas se encuentra el eslabón para el último tramo.

En el último segmento -el cuarto- se analizó la producción del mexicano a partir de sus escritos más recientes. Si bien mostramos que algunos de ellos tenían ya versiones previas, también se destacó el conjunto de novedades, tanto de fuentes e inspiraciones, como de una temática central: la crítica de la democracia. Ella parte de su muy específica lectura de El Capital y pasa hacia un conjunto de registros que no se encontraban previamente explicitados. Mostramos ahí la persistencia de un conjunto de temas, como la crítica a la dialéctica o el asedio al "materialismo", desde su concepción.

Hasta el momento no existe una evaluación del papel de Olmedo como teórico en diálogo-discusión con el marxismo. Ello ha debilitado la comprensión de un fenómeno poco usual en la región latinoamericana: el desarrollo del marxismo por fuera de los cánones de la izquierda.

\section{Bibliografía}

Althusser, L. (1970). Lenin y la filosofía. México: Era.

Althusser, L. (1978). Carta de Louis Althusser a Raúl Olmedo. Historia y sociedad. (17).

Althusser, L. (1992). El porvenir es largo. Madrid: Destino.

Althusser. L. (2015). Sobre la reproducción. Madrid: Akal.

Draper, S. (2017). Por una recepción irreverente del materialismo aleatorio en México: Althusser, Navarro y el materialismo del encuentro. En M. Rodríguez y M. Starcenbaum (comp.), Lecturas de Althusser en América Latina. Santiago de Chile: Doble Ciencia.

Esquivel, M. (2015). Alberto Híjar: lucha de clases en la imaginación. México: Cisne Negro.

Gómez. N. (2017). Leíamos a Althusser. Pasado y presente del marxismo intelectual en cuba. En M. Rodríguez y M. Starcenbaum (comp.), Lecturas de Althusser en América Latina. Santiago de Chile: Doble Ciencia.

González Rojo. E. (1974). Para Leer a Althusser. México: Diógenes. 


\section{Tras las huellas del althusserianismo teórico. Una aproximación a la obra teórica de Raúl Olmedo}

Illades, C. (2018). El marxismo en México. México: Taurus.

Malamud M. (2017). Escritos (1969-1987). Santiago de Chile: Doble Ciencia.

Moulian, T. (2009). Condiciones del desarrollo político chileno. Santiago de Chile: LOM.

Olmedo R. (1967). Los mecanismos sicológicos del prejuicio racial y su expresión en los Estados Unidos. Comunidad. (8).

Olmedo R. (1968). El estructuralismo y las teorías de Althusser, Debray y Gunder Frank sobre el capitalismo actual. Comunidad. (9).

Olmedo R. (1970). Teorías sobre el subdesarrollo. Pensamiento Crítico, (36).

Olmedo, R. (1980a). Comentario a "Retórica de la falsa contradicción" de Miche Plon. En M. Monteforte (coord.), El discurso político. México: UNAM.

Olmedo, R. (1980). El anti método. México: Joaquín Mortíz.

Olmedo, R. (1983). México: economía de la ficción. México: Grijalbo.

Olmedo, R. (1983aㅜ). “A cien años de Marx”, en Excélsior, 14 de marzo de 1983.

Olmedo, R. (1983b). “A cien años de Marx: la ley histórica”, en Excélsior, 17 de marzo de 1983.

Olmedo, R. (1983c). “A cien años de Marx: la lucha de clases”, en Excélsior, 18 de marzo de 1983.

Olmedo, R. (1983d). "A cien años de Marx: la eterna compraventa”, en Excélsior, 19 de marzo de 1983.

Olmedo, R. (1983e). “A cien años de Marx: correlación de fuerzas”, en Excélsior, 23 de marzo de 1983.

Olmedo, R. (1983f). "A cien años de Marx: psicología”, en Excélsior, 24 de marzo de 1983.

Olmedo, R. (1983g). "A cien años de Marx: la teoría del valor-trabajo”, en Excélsior, 25 de marzo de 1983.

Olmedo, R. (1983h). “A cien años de Marx: la equivalencia”, en Excélsior, 26 de marzo de 1983.

Olmedo, R. (1983i). “A cien años de Marx: la división del trabajo”, en Excélsior, 28 de marzo de 1983. 


\section{Jaime Ortega}

Olmedo, R. (1983j). "A cien años de Marx: el origen del valor”, en Excélsior, 29 de marzo de 1983.

Olmedo, R. (1983k). "A cien años de Marx: el poder y la distribución”, en Excélsior, 30 de marzo de 1983.

Olmedo, R. (1983l). “A cien años de Marx: fuerza creadora”, en Excélsior, 1 de abril de 1983.

Olmedo, R. (1983m). “A cien años de Marx: intercambio desigual”, Excélsior, 2 de abril de 1983.

Olmedo, R. (2001). La universidad en la era de la globalización neoliberal. México: Comuna. 207 pp.

Olmedo, R. (2013). Democracia: máquina tautológica que produce deseo y placer de dominar y de someterse. México: UNAM.

Olmedo, R. (2015). Democracia es religión. México: UNAM.

Olmedo, R. (2016). Las democracias perdidas. México: UNAM.

Olmedo, R. (2016aㅡ. Violencia es religión: los mecanismos del pensamiento que originan las violencias. México: UNAM.

Ortiz, C. (2001). Teoría y política en la obra de Carlos Pereyra. México: PyV.

Pacheco, V. (2017). Enrique González Rojo: trabajo intelectual y revolución articulada (1972-1981). En M. Rodríguez y M. Starcenbaum (comp.), Lecturas de Althusser en América Latina. Santiago de Chile: Doble Ciencia.

Rodríguez Araujo, O. (2002), Izquierdas e izquierdismo. México: Siglo XXI.

Rodríguez, M. y Starcenbaum, M. (2017). Introducción. En M. Rodríguez y M. Starcenbaum (comp.), Lecturas de Althusser en América Latina. Santiago de Chile: Doble Ciencia.

Rolón Anaya, M. (1969). "Dos formas de estructuralismo: revolucionarios y guerrilleros", Comunidad, (10).

Starcenbaum, M. (2017aㅡ). Marxismo, estructuralismo y psicoanálisis: Itinerarios de Althusser en la cultura psicoanalítica argentina (1965-1976). En A. Popovitch (Ed.). Althusser desde América Latina. Buenos Aires: Biblos.

Recibido: 29/05/2020

Evaluado: $20 / 07 / 2020$

Versión Final: 15/09/2021 